\title{
Human Challenge Trials in Vaccine Development, Rockville, MD, USA,
}

\section{September 28-30, 2017}

Marc FD Baay ${ }^{a}$, Thomas L. Richie ${ }^{b}$, and Pieter Neels ${ }^{c^{*}}$, on behalf of HCT2 ${ }^{\#}$

a - P95 Pharmacovigilance and Epidemiology Services, Leuven, Belgium

b - Sanaria Institute for Global Health and Tropical Medicine, Rockville, Maryland, USA

c - International Alliance for Biological Standardization, Lyon, France.

${ }^{*}$ Corresponding author: Pieter Neels, International Alliance for Biological Standardization, Lyon, France, email: pieter.neels@vaccine-advice.be

Marc FD Baay-marc.baay@p-95.com

Thomas L. Richie - trichie@sanaria.com

\# Session chairs at the second Human Challenge Trials meeting (in alphabetical order):

Cavaleri, Marco - European Medicines Agency, London, United Kingdom

Chilengi, Roma - Centre for Infectious Disease Research, Zambia

Diemert, David - George Washington University, Washington, D.C.

Hoffman, Stephen L. - Sanaria Inc., Rockville, Maryland

Johnson, Robert - National Institute of Health, Bethesda, Maryland

Kirkpatrick, Beth D. - University of Vermont School of Medicine, Burlington, Vermont

Knezevic, Ivana - World Health Organization, Geneva, Switzerland

Laurens, Matthew - University of Maryland School of Medicine, Baltimore, Maryland

McShane, Helen - The Jenner Institute, University of Oxford, United Kingdom

Njuguna, Patricia - Chair of Clinical Research, Kenya Medical Research Institute-Wellcome

Trust Research Programme, Kenya

Older Aguilar, Anastazia - Bill \& Melinda Gates Foundation, Seattle, Washington

Pollard, Andrew J. - Oxford University, United Kingdom

Riddle, Mark - Uniformed Services University of the Health Sciences, Bethesda, Maryland Sauerwein, Robert - Radboud University, Nijmegen, The Netherlands

Southern, James - Developing Country Vaccine Regulators' Network, West Cape Province, South Africa

Tribble, David - Uniformed Services University of the Health Sciences, Bethesda, Maryland Wildfire, Adrian - SGS Belgium NV, Mechelen, Belgium 


\section{Abstract}

The International Alliance for Biological Standardization organized the second workshop on human challenge trials (HCT) in Rockville, MD, in September 2017. The objective of this meeting was to examine the use of $\mathrm{HCT}$, in response to the continuing human suffering caused by infectious diseases, preventable by the development of new and improved vaccines. For this, the approach of HCT could be valuable, as HCT can provide key safety, tolerability, immunogenicity, and efficacy data, and can be used to study host-pathogen biology. HCT can generate these data with speed, efficiency and minimal expense, albeit not achieving the same robust prediction of vaccine-induced protection as field efficacy as trials. Incorporated wisely into a clinical development plan, HCT can support optimization or downselection of new vaccine candidates, assuring that only the worthiest candidates progress to field testing. HCT may also provide pivotal efficacy data in support of licensure, particularly when field efficacy studies are not feasible.

Many aspects of HCT were discussed by the participants, including new and existing models, standardization and ethics. A consensus was achieved that HCT, if ethically justified and performed with careful attention to safety and informed consent, should be pursued to promote and accelerate vaccine development.

Keywords:

Human challenge trials; infection models; vaccine; efficacy; ethics; controlled human infection; controlled human malaria infection Abbreviations: AMP - auxiliary medicinal product; BAL - bronchoalveolar lavage; CDER - Center for Drug Evaluation and Research; cGMP - current Good Manufacturing Practices; $\mathrm{CHHI}$ - Controlled 
Human Hookworm Infection; CHI - controlled human infection; CHMI - controlled human malaria infection; CIOMS - Council for International Organization of Medical Sciences; DCVRN - Developing Country Vaccine Regulators' Network; DENV - Dengue virus; EMA European Medicines Agency; ETEC - Enterotoxic Escherichia coli; EU - European Union; FDA United States Food \& Drug Administration; GCP - Good Clinical Practice; HCT - human challenge trial; HMPV - Human metapneumovirus; IABS - International Alliance for Biological Standardization; IMP - Investigational Medicinal Product; IND - Investigational New Drug; RCT - randomized controlled trial; IRB - institutional review board; LAIV - live attenuated influenza virus; NAT - nucleic acid testing; PCR - polymerase chain reaction; PfSPZ Plasmodium falciparum sporozoites; QC - Quality Control; RSV - respiratory syncytial virus; TBS - thick blood smear; TFDA - Tanzanian Food and Drug Agency; WHO - World Health Organization; ZIKV - Zikavirus 


\section{Introduction}

After the successful workshop on human challenge trials (HCTs), organized by the International Alliance for Biological Standardization (IABS) in Strasbourg, France, in 2014 [1], a follow-up meeting was held in Rockville, MD, USA, September 28-30, 2017 (See appendix for the program, or visit https://www.iabs.org). The objective of this second meeting was to examine and promote the use of HCTs or controlled human infections (CHI), in response to the continuing human suffering caused by infectious diseases around the globe, which should be preventable by the development of new and improved vaccines for which the approach of $\mathrm{CHI}$ could be valuable. As the term $\mathrm{CHI}$ more closely reflects the infectious nature of the experiment, the abbreviation $\mathrm{CHI}$ will be used throughout this document, rather than $\mathrm{HCT}$.

The agenda for the meeting was based on the principle that $\mathrm{CHI}$ can provide key safety, tolerability, immunogenicity, and efficacy data supporting vaccine research, and can be used to study host-pathogen biology. Unlike large field trials, $\mathrm{CHI}$ can generate these data with speed, efficiency and minimal expense, albeit without assurance that findings will accurately bridge to the field. Often, large field trials will still be required to generate pivotal data demonstrating safety and benefit to individuals and public health to satisfy regulatory requirements for product licensure. However, such trials are expensive, may require thousands of subjects, and can take years to complete. In contrast, $\mathrm{CHI}$, despite not necessarily mimicking wild-type infection, generally involve only tens of subjects and require only a few months to complete. Incorporated wisely into a clinical development plan, $\mathrm{CHI}$ can support optimization or down-selection of new vaccine candidates, assuring that only the worthiest candidates progress to field testing. $\mathrm{CHI}$ may also provide pivotal efficacy data 
in support of licensure, particularly when field efficacy studies are not feasible, e.g. due to low attack rates.

Deliberate exposure of humans to infectious agents has been performed in the Western world since the early $18^{\text {th }}$ century, when variolation, based on historical use of this approach in the Orient and Middle East, was tested for the first time in the UK as a prevention for smallpox. Early $\mathrm{CHI}$ studies, however, sometimes lacked adequate ethical justification. For example, some were performed in vulnerable populations, e.g., orphans and prisoners, and did not fully adhere to important ethical principles, as these had not been formally elucidated and their centrality to human research was not promulgated until the publication of the Belmont report in 1979 [2].

Since the 1970s, major changes have occurred in public opinion about $\mathrm{CHI}$ and the ethical and regulatory context in which $\mathrm{CHI}$ are performed. Studies now generally enroll healthy, non-disadvantaged volunteers from the local community, in compliance with ethical standards, assuring informed consent, under the oversight of institutional review boards (IRBs), and, increasingly, within the purview of regulatory authorities such as the United States Food \& Drug Administration (FDA) and European Medicines Agency (EMA). Participants in $\mathrm{CHI}$ are well-informed of the risks, free to choose or decline participation, perceive (and are motivated by) societal benefit, and are compensated for their time. Key elements supporting the effective use of $\mathrm{CHI}$ are the development of well-characterized and quality-controlled challenge strains, standardized procedures for inoculation, formally approved clinical protocols, pre-selected outcome measures, dedicated human challenge facilities, access to adequate numbers of volunteers, and regulatory endorsement of the 
value of the data obtained. A landmark was achieved in 2016 with the licensure of the cholera vaccine Vaxchora ${ }^{\mathrm{TM}}$ by the FDA, based on demonstration of safety in healthy volunteers, and efficacy data obtained from $\mathrm{CHI}$, supplemented by safety data collected in larger study populations.

Many aspects of $\mathrm{CHI}$ were examined by the participants who convened in Rockville. Altogether, the program comprised of 55 presentations, 5 panel discussions, and 3 pointcounterpoint debates. A consensus was achieved that $\mathrm{CHI}$, if ethically justified and performed with careful attention to safety and informed consent, should be pursued vigorously and with expanded applications aiming to promote and accelerate vaccine development. An important outcome was the proposal that vulnerable populations suffering a heavy disease burden might be considered for inclusion in $\mathrm{CHI}$ that significantly advance vaccine development, particularly when other populations cannot provide adequate surrogate data. This is a way to address the paucity of data in populations where vaccines are most needed, especially in endemic settings, stressing, however, the need to develop shared and appropriate ethical guidelines. This and additional concepts are listed for further exploration in future meetings. The momentum and enthusiasm generated by the meeting are being harnessed by IABS, which is planning a third HCT event in Oxford, UK, in 2019.

\section{Regulatory framework for Human Challenge Trials}

The FDA requires that $\mathrm{CHI}$ are conducted under an Investigational New Drug (IND) application [3]. The purpose of regulatory oversight of human challenge models is to assure the safety and rights of subjects, by risk assessment, risk mitigation and risk communication. 
Furthermore, IRB review and informed consent are necessary. Clinical considerations for $\mathrm{CHI}$ may include the exclusion of children, elderly, pregnant women, immunocompromised individuals and other vulnerable populations; pre-challenge testing to ascertain health of volunteers; and post-challenge testing for carrier status. Using $\mathrm{CHI}$ to support vaccine effectiveness, limitations of the challenge model and feasibility of conducting a field efficacy study should be considered. Upon licensure, limitations of the model or uncertainties related to vaccine effectiveness may be reflected in prescribing information. Nevertheless, the FDA views $\mathrm{CHI}$ as potentially good tools, comparable to early phase clinical trials. Therefore, full adherence to current Good Manufacturing Practices (cGMP) appropriate to Phase 3, including validation of associated processes and assays, may not always be necessary, as long as good science, reliable assays, and sound scientific judgement are used. The EMA does not approve $\mathrm{CHI}$, nor randomized controlled trials (RCTs), as review and approval are tasks of the individual Member States. Consequently, a universally agreed upon understanding on the regulatory requirements for the review and approval of $\mathrm{CHI}$ is lacking, but clearly needed.

The African experience was discussed, using the Tanzanian Food and Drug Agency (TFDA) as an example; the agency was asked in 2012 to review the protocol for the first proposed performance of a controlled human malaria infection (CHMI) in Africa. Guidelines and policies had to be newly developed, providing a positive learning experience. Tanzania was the first African country to perform CHMI [4], and soon thereafter became the first African country to use $\mathrm{CHMI}$ to measure vaccine efficacy (Jongo, AJTMH, in press). The TFDA closely monitored the $\mathrm{CHMI}$ studies to ensure that the rights, safety and well-being of the participants were observed, and that data credibility was maintained. This experience 
helped to empower drug authorities in three other African countries to approve CHMI studies.

In $\mathrm{CHI}$ there is a focus on finding correlates of protection, however, these are sometimes elusive. When the best correlate of protection is T-cell-based, tests are not easy to standardize between labs. Because the efficacy results of $\mathrm{CHI}$ may not bridge to field efficacy, usually there is still a need to test efficacy in RCTs. Although CHI may have a value in streamlining clinical development, general acceptance and explicit guidance from regulators is not available. It is therefore imperative for manufacturers to have discussions with regulatory authorities at an early stage of vaccine development, before any studies are initiated, exploring the use of $\mathrm{CHI}$, along with the merits and considerations of such an approach for the specific product and condition.

\section{Human challenge trials guidelines}

In order to facilitate research, development and implementation of vaccines and biologicals, the World Health Organization (WHO) has prepared written standards, recommendations and guidelines, as well as measurement standards. The aim is to provide key principles for evaluation of biologicals, while leaving room for regulatory agencies to formulate additional requirements. Where possible, the WHO will assist in the implementation of the guidelines through workshops, training and advisory groups. Similarly, scientific and regulatory considerations have been established, to help in ethical and regulatory issues. In a consultative process, the considerations are discussed with the various stakeholders, and adapted where needed before setting the standards, which are then revised and/or updated at least every 10 years. Finally, as there is no enforcement mechanism, individual countries may set their own standards. 
The Developing Country Vaccine Regulators' Network (DCVRN) was established in 2006, supported by WHO, and currently has representatives from 9 countries around the globe. It is a network for mutual assistance, extracting procedures from established regulatory authorities. So far, no $\mathrm{CHI}$ have been performed in the DCVRN countries, with the exception of the attenuated rotavirus vaccine challenge of infants in South Africa [5]. While the DCVRN members recognize the potential advantages of $\mathrm{CHI}$, it is expected that the regulatory authorities would be reluctant to permit $\mathrm{CHI}$, due to concerns over the safety of participants; the potential risk to spread the infection; control of (the reproducibility of) the challenging dose; fear of the public perception of $\mathrm{CHI}$; and the need to confirm $\mathrm{CHI}$ results in phase 3 clinical trials. Considering coercive (excessive) payment for participation, the aim should not be equal payment, but equal spending power. In DCVRN countries, quite substantial payment is provided for participation in phase 1 trials, and payment for $\mathrm{CHI}$ would be expected to be of similar level. A formula for the level of payment was developed, aiming for a minimum payment, which is supplemented if necessary (e.g. in the case of serious adverse events [SAEs], including hospitalization).

In $\mathrm{CHI}$, standardization is crucial, as well-designed, well-performed studies with little in common are much less convincing. While sharing strains is essential, the potential drawback of using a limited number of strains is that they may not be representative of the diversity in nature, or there may be antigenic change/drift in the challenge organism. $\mathrm{CHI}$ have not reached their full potential to support vaccine and drug development, due to limitations in $\mathrm{CHI}$ development and utilization, and lack of alignment of the available models with the candidate vaccines to be evaluated. To further the use of $\mathrm{CHI}$, PATH, supported by the Bill \& 
Melinda Gates Foundation, has established a CHI Consortium to enable a network of investigators using human infection models to: facilitate the use of state-of-the-art technologies, and to promote standardization of specimen collection and analysis.

Performing $\mathrm{CHI}$ is a way to learn and test, while minimizing the number of subjects. Risks and benefits need to be carefully balanced, recognizing that there will generally be no shortterm benefits for research subjects. Performing a post-CHI questionnaire is optimal to learn from the participants' experience, bearing in mind that $\mathrm{CHI}$ are a fit-for purpose model, which differs from real world clinical practice.

\section{Quality standards for Challenge Strains}

The FDA demands that for the production of challenge strains, Chemistry Manufacturing and Controls guidance applies in regard to strain characterization, purity, potency, and stability/viability. Facilities for production of challenge agents should comply with cGMP appropriate to the phase of the clinical study. In the case of bacterial and parasitic diseases, safety of the challenge strain is ensured by verification that infection can be terminated by treatment, and the absence of genetic alterations. The challenge strain should not contain other pathogens. The appropriate dose of the challenge strains must be determined: a higher dose may lead to pathology; a lower dose may not lead to an informative study. In the European Union (EU), Directive 2001/83/EC applies to medicinal products for human use intended to be placed on the market. This Directive does not apply to medicinal products intended for research and development trials. On the other hand, the directive states that an Investigational Medicinal Product (IMP) is a pharmaceutical form of an active substance or placebo being tested or used as a reference in a clinical trial. The new 
Regulation 536/2014 on clinical trials describes auxiliary medicinal products (AMP) to include medicinal products used for background treatment; (pharmacological, not biologic) challenge agents; rescue medication; or products used to assess end-points in a clinical trial, but not concomitant medications. It remains unclear whether a challenge pathogen preparation can be considered as a medicinal product, especially as no definitive position has been taken yet at the Member State level. It is suggested that an application dossier should be prepared containing information on biological properties, qualification and characteristics, labelling, manufacturing, control, traceability, storage, return and destruction of the substance/AMP. Furthermore, a description of pharmacovigilance tasks is required. Challenge material should be manufactured according to GMP or to an equivalent standard, to ensure an appropriate quality management system for the main tasks, with a qualified person to ensure compliance with requirements. Clinical trial and $\mathrm{CHI}$ applications are within the remit of Member States, leaving room for different interpretation among EU Member States. Two guidelines cover the applicable provisions:

EMEA/CHMP/BWP/534898/08 - Requirements for quality documentation concerning biological investigational medicinal products in clinical trials, and EMEA/CHMP/BWP/398498/2005 - Virus safety evaluation of biotechnological investigational medicinal products.

Similar to clinical trial sites, $\mathrm{CHI}$ sites can expect to be audited at some point, whether as a routine visit, as pre-approval following submission of a new product application or for a specific cause. To be optimally prepared, a clear and comprehensive Quality Control (QC) plan, data management plan and quality records need to be available at all times. 


\section{Development of Challenge Strains}

To be informative of protective efficacy against natural infection, $\mathrm{CHI}$ need to use adequate pathogen strains. Homologous challenge is defined as challenge with an organism that is genetically (or immunogenically) identical to the vaccine strain, while heterologous challenge uses an organism that differs immunogenically from the vaccine strain. Finally, strains used in challenge trials should be representative of field strains, particularly those originating from the same geographic region. Characterization of the genome sequence of five Plasmodium falciparum strains, spanning South America, Africa and South-East Asia, showed that NF54 is nearly identical to 3D7, whereas 7G8, NF166, and NF135 showed significant differences in coding regions and pre-erythrocytic antigens [6]. Furthermore, the strains are representative of the populations from which they originated.

Sanaria Inc., a biotechnology company developing malaria vaccines, has pioneered the manufacturing of aseptic, purified, cryopreserved, cGMP-compliant, $P$. falciparum sporozoites (PfSPZ) for parenteral injection. The portfolio includes PfSPZ vaccine, comprised of radiation-attenuated sporozoites; two strains of PfSPZ challenge, comprised of fully infectious sporozoites for inoculation of volunteers for $\mathrm{CHMI}$ or immunization under drug cover (PfSPZ-CVac); and PfSPZ-GA1, comprised of genetically attenuated sporozoites. The products are cryopreserved in liquid nitrogen vapor phase, and are stable for at least four years, with potency confirmed by laboratory assays and by documenting infectiousness when administered to humans. Since October $2010,>800$ volunteers have participated in CHMI trials in the US and 9 countries in Europe and Africa using PfSPZ challenge injected parenterally. 
An alternative to using $\mathrm{CHI}$ for assessing the efficacy of subunit vaccines is the development of "humanized" murine pathogens that can be studied in mice. An example is chimeric Plasmodium berghei parasites engineered to express the circumsporozoite proteins of the human parasites $P$. falciparum or $P$. vivax $[7,8]$. These models allow the use of mice for testing novel CSP-based vaccine candidates, such as a self-assembling protein nanoparticlebased malaria vaccine [9], or monoclonal antibodies binding to the vaccine antigen [10], potentially defining new protective epitopes. Specific chimeras can be engineered to study other antigens, such as TRAP or CelTOS, and guidelines have been published [11].

Evidence of egg-adaptation was already shown for influenza in the 1980s [12, 13], which changes antigenicity and receptor use, potentially leading to reduced attack rates. Furthermore, pre-2013 H3N2 challenge agents are haemagglutinating, whereas the currently circulating strains are non-haemagglutinating [14]. Challenge agents should have the following properties: short, acute, self-limiting infection with rapid incubation; moderate morbidity in healthy populations; overtly symptomatic (fever and myalgia), but with limited sequelae; predominance as a circulating strain with global distribution; epidemic or even pandemic potential; broad antigenic diversity; potential to be cultured in eggs or cell lines; and, evoking a measurable curative (or prophylactic) immune response to infection. To identify and isolate a new challenge agent, relevant source populations need to be determined, using community surveillance programs to inform choices. Established, predominant or circulating strains will offer greater relevance to study data than minority or uncharacteristically resistant serotypes, whereas newly emergent, pandemic strains may offer unacceptable risks of adverse events or sequelae, including a high case fatality rate. To preserve viral fitness of the challenge strain, seed-stocks from proven, virulent strains must 
be used. The presence of mutations in attachment proteins must be excluded. Similarly, surface proteins must be checked for phenotypic changes. An influenza challenge strain was developed using GMP, for a 'First in Human' phase 1 trial. Scientific advice was obtained from the Belgian Health Authority to validate the development/manufacturing plan. The H3N2 influenza virus was isolated from a 14-year-old male. The manufacturing approach was based on a combination of EMA and FDA vaccine guidelines. The challenge agent was manufactured as a solution for intranasal use by a contract manufacturer, which underwent a GMP classification audit. A preclinical study was performed in ferrets. After approval, the challenge agent was used in a dose escalation study to determine the safety and attack rate in healthy volunteers (EudraCT Number: 2016-002737-29). The objective was to determine the dose with an attack rate of $>60 \%$. The process of preparing a challenge strain took 1.5 years.

Most dengue infections are asymptomatic or infected persons do not present for care. In symptomatic infection, fever, rash, headache, myalgia, and retro-orbital pain may occur, while severe dengue may lead to hemorrhage, thrombocytopenia, vascular leak, and shock, and can be fatal. A 30-base pair deletion in the 3-terminal region of the viral genome of DENV2 and DENV3 led to challenge strains inducing viremia in all healthy volunteers, while none developed fever, elevated liver function tests or signs of vascular leak. A similar construct for DENV1 was less effective. Studies showed that the vaccine completely protects against viremia and rash after homologous challenge [15].

\section{Development of New Challenge Models}

Progress of development of several new challenge models was presented: 
- Schistosoma. A CHI has been developed, based on the production of male cercariae under GMP conditions. A dose escalation study is being performed, to find the optimal dose. Most common adverse events were rash, headache and fever, which could last for several weeks, and rarely were grade 3. A dose-related increase of CAA could be observed, useful as a diagnostic marker, and to show effective treatment with praziquantel.

- Mycobacterium tuberculosis. Treatment of tuberculosis can be long, up to 6 months for drug-sensitive strains, and 2 years for multidrug-resistant strains, potentially having serious side effects. Therefore, on ethical grounds, we cannot deliberately infect people with M. tuberculosis. Work to develop an attenuated strain with a kill switch is underway. In such a strain, a control system is needed which eliminates all bacteria at the end of the study, and a detection system that can measure the bacterial load in the lungs in a non-invasive or minimally invasive way. In the current absence of a mycobacterial strain that fulfils these conditions, BCG, an attenuated replicating strain of $M$. bovis, licensed for human use, can be used as a challenge organism. BCG vaccination protects against subsequent BCG challenge in mice [16], cattle [17] and non-human primates (Harris et al, in press). An initial study used intradermal challenge of humans with BCG [18], which is not the natural route of infection. Therefore, an aerosol BCG challenge model is now being developed, and a dose escalation study is being performed, with no safety concerns encountered so far.

- Rotavirus. The current rotavirus vaccine leaves a huge residual burden, as it is too expensive and less effective in low- and middle-income countries (LMICs). No clear correlate for protection has been established. A subunit protein vaccine may provide 
superior efficacy, but placebo-controlled efficacy trials are difficult to justify. The current live-attenuated oral vaccine can be used as challenge organism in a $\mathrm{CHI}$. Initial dose-escalating studies on a monovalent subunit vaccine have successfully been performed, with promising signs of immunogenicity [19]. Safety, immunogenicity and efficacy of the subunit vaccine to protect against shedding of the attenuated virus in stool samples were investigated in a $\mathrm{CHI}$ performed in infants [5]. Good IgA and IgG response rates were observed, and a significant reduction in viral shedding. Further studies, with a wider range of dose levels will be performed. Nevertheless, it is unclear whether impact on shedding relates to clinical impact. - Zika virus. Congenital Zika virus (ZIKV) syndrome can occur regardless of the timing of maternal infection, and severity of disease in the mother is not associated with the occurrence of ZIKV congenital syndrome in the infant [20]. Hence, a ZIKV vaccine that prevents infection is required. Currently, 45 candidate vaccines have been described, showing the need for down-selection. Well-controlled $\mathrm{CHI}$ may help to answer a number of questions: can sterilizing immunity be induced, and what would be its duration; what is the effect of pre-existing flavivirus immunity on ZIKV protection; can passively-transferred antibody protect against ZIKV infection? Furthermore, a CHI can identify correlates of protection and inform the development of public health guidelines related to transmission of ZIKV. However, the NIH ethical review committee decided against the use of a ZIKV CHI [21], based on the uncertainty of the risk posed to the volunteers, as well as to third parties (fetus, sexual contacts, etc.), as the virus may spread outside the research environment. Furthermore, the duration of protection needed is uncertain. Finally, the societal value of a ZIKV CHI was deemed uncertain, as there may be alternatives available, and the use of $\mathrm{CHI}$ 
would not preclude the need for field trials at a later stage. However, the applicants felt that this decision was based on some key misunderstandings: the value of $\mathrm{CHI}$ to down-select candidate vaccines; the wealth of information that can be obtained from $\mathrm{CHI}$ on ZIKV transmission other than maternal-fetal; and the perception that a ZIKV disease model would be needed, where a ZIKV infection model would suffice. Additionally, Zika virus transmission has decreased substantially, making it unlikely a traditional efficacy study will able to be conducted. A ZIKV CHI could assess efficacy in this circumstance, a benefit the review committee acknowledged. Risks associated with a ZIKV CHI are being evaluated, but scientific evidence suggests that appropriate risk mitigation (e.g., excluding pregnant volunteers or those with a pregnant partner; conducting in-patient studies; providing monitoring to partners; enrolling younger volunteers, ensuring availability of diagnosis and treatment for all volunteers) will allow the study to proceed in a safe and ethical manner.

- Bordetella pertussis. In the case of pertussis, despite the availability of vaccines, 161,000 deaths occur per year worldwide [22]. In the developed world, the number of pertussis cases is increasing, related to breakthrough cases, suggesting a new vaccine that prevents colonization may be required. Pertussis colonization and progression to disease in vaccinated individuals are not fully understood, and $\mathrm{CHI}$ might help, as field studies are hard to perform given that incidence of pertussis is low and varies over time and geographically. Because $B$. pertussis is an air-borne pathogen, containment is important, yet difficult to achieve. Furthermore, disease in adults is generally mild, but can be severe, even fatal. Antibiotic intervention needs to be administered early. Although children are the primary target population, $\mathrm{CHI}$ in adults are useful to explore correlates of protection that are adaptable to the 
pediatric population. Dose-finding studies are currently being performed in the UK and Canada.

\section{Refining and Optimizing Existing Controlled Human Infection Models}

Similarly, progress on refinement and optimization of several existing challenge models were presented:

- Controlled Human Hookworm Infection (CHHI). Studies are needed for vaccine validation. A vaccine based on a combination of recombinant proteins has been developed, and phase 1 trials have been performed, however, phase 3 trials can only be done after proof of concept has been established. Previous $\mathrm{CHHI}$ have been done in the 1980s, showing that infection is well-tolerated, has a predictable time to patency, with minimal AEs, both in numbers and severity, although skin rash can be severe at higher dose. Finally, hookworm infection can be cured with albendazole. A dose of 50 larvae proved sufficient to infect $90 \%$ of subjects. This dose will be used, 28 days after completion of vaccinations, to assess protection against infection.

- Enterotoxic Escherichia coli (ETEC). The CHI for the evaluation of ETEC vaccines has undergone several refinements [23]. The use of an overnight fast led to a reduction of the challenge dose by 1-2 logs, while retaining comparable rates of symptoms. Lyophilization of the challenge organism led to a vast simplification and standardization of the dose preparation protocol. Finally, to standardize endpoint definition, a disease severity score system was developed [24], facilitating the differentiation of disease profiles when equal attack rates were obtained.

- Malaria. Plasmodium nucleic acid testing (NAT) can be used to replace thick blood smear (TBS) testing in CHMI trials. NAT is routine practice in most laboratories, and 
analytical validation shows a good linear range, but clinical validation is necessary.

NAT assays are positive 2.9-3.6 days before TBS. Because of earlier detection, treatment can be started earlier, so no in-patient phase is necessary, making the trial more cost-effective. NAT reduces symptoms, as illness is proportional to parasite density, without eliminating safety endpoints. While the goal is to obtain sterile protection, partial protection must not be overlooked. Work is ongoing to obtain Biomarker Qualification with the FDA Center for Drug Evaluation and Research's (CDER) Drug Development Tool Program.

\section{Human Challenge Trials to Support Licensure}

For some diseases, field trials of vaccines are nearly impossible due to the low incidence rate of the disease. So far, only one example exists where $\mathrm{CHI}$ supported licensure (cholera, described below), however, there are several other organisms where $\mathrm{CHI}$ might help to support licensure:

- Cholera, a non-invasive diarrheal disease, caused by the ingestion of Vibrio cholerae, leads to life-threatening loss of fluid and electrolytes induced by the cholera toxin subunit $A$. The oral live attenuated cholera vaccine CVD 103-HgR, not producing cholera subunit $A$, but producing immunogenic subunit $B$, was recently approved in the US for travelers, based on challenge studies, as a field (efficacy) trial in travelers was not feasible due to the low and unpredictable rate of cholera infections. The challenge studies showed that the vaccine had an efficacy $>90 \%$ after a single dose [25]. The serum vibriocidal response correlated with protection against cholera, enabling bridging studies to other populations. Safety and immunogenicity were confirmed in field trial of $>3,000$ subjects. 
- Malaria. Although the number of deaths due to malaria have halved from 2000 to 2015 , due to the use of bed nets, new drugs and indoor insecticide spraying, the number is plateauing, demonstrating the need for a vaccine preventing infection. A vaccine based on radiation-attenuated Plasmodium falciparum Sporozoites (PfSPZ) is available. Though the vaccine's efficacy can be measured in traditional double-blind, placebo-controlled RCTs, conducted in endemic areas, these are laborious, timeconsuming and costly. The use of $\mathrm{CHMI}$ could enable investigation of efficacy more quickly, less expensively, with limited-sized study populations. Infection can be achieved by mosquito bites or by direct venous inoculation. The vaccine provides a high short-term protection against homologous (90\%) and heterologous (80\%) infection, with long-term protection against homologous infection between 55-65\%, with room for improvement based on regimen optimization [6]. Long-term protection (i.e. >6 months) against heterologous infection varied from 29-54\%, but increased with higher antigen dose $[6,26]$. However, naturally acquired immunity significantly impacted the short- and long-term protection level: protection was $92.3 \%$ and $70 \%$ in US adults, respectively, compared to $20 \%$ and $0 \%$ in Tanzanian adults.

- Norovirus. This virus is highly contagious, environmentally stable, and infection can be fatal in the elderly and the very young. No animal model exists, therefore, $\mathrm{CHI}$ have been used to show the effect of norovirus vaccines before field trials. When, after vaccination with GI.1 monovalent vaccine or placebo, subjects were challenged with norovirus GI.1, limited protection against infection, but a significant reduction in illness was observed [27]. Similarly, the GI.1/GII.4 vaccine showed a reduction in 
illness of $52-100 \%$, the reduction was most pronounced against more severe disease $[28]$.

- Shigella. Although the global burden of disease has declined over the last decades [29], it remains high, and shigellosis is particularly of concern to the US military [30]. As few as 10 organisms can cause disease, presenting a significant risk in environments where Shigella may be present. A Shigella $\mathrm{CHI}$ is available, with consistent attack rates and correlates of protection. Two challenge strains are available: S. flexneri $2 a$ and S. sonnei. Attack rates of S. flexneri $2 a$ are high for diarrhea, but low for dysentery [31]: a comparatively safe challenge strain. Initial results from a Shigella $\mathrm{CHI}$ after vaccination with Flexyn2a or placebo showed protection against more severe diarrhea, early administration of antibiotics, and intravenous fluids (unpublished data, Clinicaltrials.gov: NCT01618591).

\section{Advancing the frontiers of human challenge research: the typhoid example}

Every year, 22 million new cases of enteric fever caused by typhoid are estimated to occur, although they frequently go undetected due non-specific symptoms and lack of accurate diagnostics. There is a specific issue of increasing antimicrobial resistance in S. typhi, and so there is an acute need for improved control measures, such as better diagnostics and vaccines. The Vi polysaccharide vaccine shows an efficacy of $70 \%$, but this decreases to $50 \%$ after three years [32]. A conjugate vaccine showed better results but only limited data are available. As no good animal model is available, $\mathrm{CHI}$ could provide valuable information by bridging polysaccharide vaccine data to conjugate vaccine data. In a typhoid $\mathrm{CHI}$, it was necessary to screen 710 people to enroll 100 participants; many of the otherwise eligible persons had previously received the vaccine. In this $\mathrm{CHI}$, healthy adults ingested S. typhi and 
were closely monitored in an outpatient setting for 2 weeks. Antibiotic treatment was initiated if typhoid was diagnosed (by either fever or positive blood culture criteria) or, if not diagnosed, at day 14 . An attack rate of $55-65 \%$ was found. Overall, challenge was well tolerated, although $10 \%$ of participants developing at least one severe sign or symptom [33]. Using CHI, MOZHO9 and Ty21a vaccines both protected against bacteremia [34]. With a definition of typhoid as a positive culture after high fever (>38C), the polysaccharide conjugate vaccine efficacy was $87 \%$. The antibody response to the conjugate vaccine was higher than to the polysaccharide vaccine, and antibody levels persisted until month 7. Bactericidal activity is induced by vaccination, important for controlling the infection, leading to fewer symptoms, but not preventing infection [35]. A model has also been developed to look at Salmonella paratyphi $A$ infection and, furthermore, in a heterologous re-challenge study: individuals with previous S. typhi exposure were not protected against rechallenge with S. paratyphi, but did show reduced overall attack rates, and a delayed time to diagnosis. The use of '-omics' technologies on CHI-derived samples would make it possible to obtain a better understanding of the pathogenicity, to drive diagnostic and therapeutic development, and to find correlates of protection. Finally, as $\mathrm{CHI}$ can only be performed if volunteers are available, an effort was invested into improving the participant's experience, looking at motivation, study experience, and satisfaction with reimbursement level.

\section{What can we learn about human immunity from Human Challenge Trials?}

The level of detail obtained with a $\mathrm{CHI}$ is dependent on the organism under investigation, prior knowledge available on this organism, and how well CHMI reproduces the natural infection and predicts drug or vaccine efficacy. In more complex organisms, such as Campylobacter jejuni, $\mathrm{CHI}$ offer unparalleled opportunities to advance human immunology, 
especially if re-challenges are done, but basic immunologic data are needed from natural disease populations before a $\mathrm{CHI}$ can be started. Furthermore, minimal standardization of assays would permit use of historical controls, and functional immune assays would facilitate discovery of correlates of protection. With a systems biology approach, statistics, modeling, and bio-informatics will become increasingly important and analytic packages must meet these challenges.

Respiratory syncytial virus (RSV) is a leading cause of respiratory infections in children resulting in 100,000 deaths per year, globally [36]. No effective RSV vaccine exists due to several hurdles, including: the lack of long-lived immunity, poor understanding of the correlates of protection, and the diversity of needs and considerations among the various high-risk populations. In an RSV CHI, the attack rate was 55\%, with two-thirds developing symptoms [37]. Peak viral load is detected later compared to influenza, and serum neutralizing antibodies do not predict infection, whereas nasal $\lg \mathrm{A}$ seems to be a correlate of protection. However, anti-RSV antibodies are short-lived, and no IgA memory B cells are induced. Similarly, CD8+ memory T cells in the blood do not correlate with protection, whereas RSV-specific memory T cells in bronchoalveolar lavage (BAL) correlate inversely with symptoms and viral load. This suggests different layers of protection against RSV infection and/or disease: pre-existing protective/immune mechanisms in the form of secretory IgA, and mucosal immunity by resident memory T cells. These data, obtained in adults, are challenging to extrapolate to infants, a high-risk population for RSV.

Norovirus has an incubation period of 24-48 hours, starting with nausea and abdominal cramps, followed by vomiting and/or diarrhea. Symptoms typically resolve in 1-3 days, but 
can be prolonged in the young, elderly and immunocompromised people. In a norovirus $\mathrm{CHI}$, 40 subjects were enrolled, 23 of whom were secretor-positive. Of the secretor-positive volunteers, $70 \%$ got infected (versus 1 secretor-negative, or $6 \%$ ), 35\% had diarrhea, $30 \%$ vomited ( $6 \%$ secretor-negative), $61 \%$ shed the virus (6\% secretor-negative) and $52 \%$ seroconverted [38]. This $\mathrm{CHI}$ shows promise for the evaluation of vaccines and antiviral drugs, whereas rechallenge studies can provide insight into immune correlates of disease.

A pneumococcal $\mathrm{CHI}$ has been developed, in which $>850$ volunteers have been challenged, and two vaccines tested. While subjects were completely protected against carriage after homologous rechallenge [39], they were only partially protected after heterologous rechallenge [40]. It was shown that anti-capsular IgG, mediating agglutination of bacteria, correlated with protection against carriage [41]. Co-infection, as mimicked by injection of live attenuated influenza virus (LAIV), led to significantly higher pneumococcus levels, and altered immune mechanisms controlling carriage, especially recruitment of monocytes to the nasal mucosa. Pro-inflammatory cytokines were increased after LAIV, and increases were higher in volunteers who went on to carry pneumococcus.

While the subunit malaria vaccine RTS,S/AS01b has been tested in a phase 3 trial, it showed poor efficacy against clinical disease, and will not meet the goal of malaria eradication [42]. An alternative strategy to developing a protective subunit vaccine would be to define other antigens expressed during the pre-erythrocytic or asexual blood stages. However, neither CHMIs nor RCTs can be performed on hundreds of candidate vaccines each composed of a different candidate antigen. One approach would be to look at variability in growth rate in semi-immune volunteers as a function of antibody responses. The Kenyan coastal population 
includes those who have an active infection, those previously infected and possessing a degree of naturally acquired immunity, and those without history of parasite infection. These individuals can be subjected to $\mathrm{CHMI}$, and then the growth rates of $P$. falciparum challenge strain in the blood can be correlated with their pre-existing immune responses to multiple antigens simultaneously. The most promising antigens can then be used to develop vaccines, that can be further tested by CHMI before progressing to RCTs.

Human metapneumovirus (HMPV), a paramyxovirus first described in 2001 [43], is a frequent cause of viral acute respiratory illness in young children. Older adults are at an increased risk for lower respiratory illness and hospitalization related to HMPV, and the virus triggers asthma exacerbations. A recombinant HMPV challenge strain was developed by substitution of four nucleotides in the SH gene, to reduce adventitious mutations that occur during passage. The stabilized challenge strain, rHMPV-SHs, was indistinguishable from wildtype virus as shown by its growth kinetics and its ability to infect animal models [44, 45]. An initial $\mathrm{CHI}$ was performed to determine the frequency of infection, the kinetics of viral shedding and the frequency and severity of disease. The virus was infectious, and detection of virus peaked at day 7 , both by culture and by polymerase chain reaction (PCR). Peak viral shedding was related to symptoms, with $50 \%$ of volunteers experiencing mild to moderate upper respiratory tract symptoms [46]. Blood samples obtained at day 28 after challenge showed that the virus was poorly immunogenic.

The abundance of a given pathogen is critical for the development of disease. The incubation period is the time needed for a pathogen to reach the necessary abundance to cause disease. However, this is affected by the abundance of other bacteria in the system. Hence, 
appropriate counting of organisms is necessary. Four methods exist, each with its own features: quantitative culture, which is expensive and labor-intensive; quantitative PCR, specific for the target organism; 16S rDNA sequencing, detecting all taxa in a sample; and metagenomic sequence analysis, which is expensive, specific for the target organism, and uses only a fraction of the data generated. Regardless of the method used, thresholds are required for disease causality.

\section{Dengue $\mathrm{CHI}$ : model of infection or disease?}

In the dengue infection model, infection is the endpoint, verified by recovery of infectious virus from the blood, by PCR or culture. This is a reproducible endpoint requiring low participant numbers, is safe and ethical, with minimal risk to participants. The challenge strains used are recombinant viruses that were not attenuated compared with their wildtype parent viruses. They cause low viremia and mild disease. $3 \log _{10}$ PFU induced viremia in more than $80 \%$ of the subjects, rash in $80-100 \%$ of subjects, but did not induce fever or liver function abnormality, allowing performance of studies on an outpatient basis. Based on this model, efficacy of protection against viremia could be shown with $<10$ subjects per group, whereas protection against rash would need 20 subjects per group. This model can be used to evaluate both vaccines and therapeutics against dengue, although DENV 1 and 4 challenge strains are currently lacking.

In a disease model, an endpoint of viremia in combination with fever and two symptoms (headache, myalgia, rash, eye pain, bone pain, joint pain, abdominal pain, muscle pain, nausea/vomiting, liver function tests, leukopenia, thrombocytopenia) is considered more informative. Taking every measure to secure the safety of the volunteers, including a Data and Safety Monitoring Board, a phase 1 dose escalation study is being performed, to 
investigate how much disease is acceptable as risk. The lowest dose has been given to 6 volunteers, of whom one was hospitalized for dengue (and one more for influenza). Such a disease model will be essential in down-selecting potential dengue vaccines, ultimately protecting volunteers in phase 3 studies from being exposed to non-effective vaccines, and will provide information on the pathogenesis of DENV infection.

\section{Safety in human challenge trials}

When setting up an $\mathrm{CHI}$, risk mitigation has to take place at multiple steps: the host, the pathogen and the environment. To be amenable for a $\mathrm{CHI}$, a disease must lead to tolerable discomfort, and effective treatment of the disease must generally be available unless the agent is intrinsically self-limiting, and rapid recovery should be the norm. Precise endpoints must be defined, so treatment can be initiated once these are met. The infection should not lead to persistent illness, or transmission to others. Notifications may be required to the general practitioner, the employer, and close household contacts. As healthy people volunteer to participate, they are prepared to take limited risk. The risks must be minimized, while maximizing the benefit.

Concerning the product safety, a CHI will provide a limited safety database, which will need supplementation with phase 3 trial data to be able to detect uncommon AEs. The pathogen needs to lack known virulence factors associated with severe clinical consequences [47]. Environmental considerations include the choice of ward (closed ward versus clinic-based), infection control measures, and availability of care for acute complications and longer-term sequelae (at least 6 months of active follow-up). Finally, the trial unit staff needs to be well prepared, and aware of the risks, with detailed cleaning instructions, including a sequence of cleaning, with logs, and adequate disposal of infectious waste. 
While $\mathrm{CHMI}$ are generally safe, some concerns exist, especially in regard to cardiac events. A first case of minimal cardiac damage with increased troponin-T levels was observed in 2007. The study was put on hold, monitoring intensified, including standard testing of troponin-T, and family history of cardiac disease was added as an exclusion criterion for future malaria CHMI. In 2013, a case of acute myocarditis was observed in an asymptomatic subject with elevated troponin-T, detected by active surveillance. In 2014, another case of acute myocarditis was diagnosed based on increased troponin-T levels. The event was deemed to be probably related to CHMI by the study's Safety Monitoring Committee. The IRB wanted to know whether there was long-term damage, whether there was any insight on causality and mechanism, and how these events could be predicted and prevented. The expert opinion was that, based on low frequency and transient nature of the complication, without longterm sequelae, myocarditis can be seen as an acceptable risk. No marker is available to predict cardiac events. In one study, intensified laboratory screening showed liver enzyme abnormalities in $60 \%$ of volunteers, which normalized in 35 days. Again, causality was unclear, with $\mathrm{CHMI}$, medication or use of alcohol as potential factors. In conclusion, the occurrence of three separate cardiac SAEs led to additional inclusion and exclusion criteria, increased active monitoring for evidence of myocarditis by laboratory testing, and earlier treatment after challenge. The cardiac risk was included in the information sheet and carefully described during meetings with volunteers.

Due to the complexity of benefit/risk assessment, a massive framework has been built around vaccines, clinical trials, licensure, and the life cycle of products, which is generally also true for $\mathrm{CHI}$. Risk management can be achieved by three steps: risk identification; risk 
assessment; and risk mitigation. As a rule of thumb: the more attenuated the challenge organism, the less informative the $\mathrm{CHI}$; conversely, the more risk there is for subjects in terms of disease acquisition and potential sequelae, the more informative the $\mathrm{CHI}$. However, if no animal models are available, $\mathrm{CHI}$ are needed. The data have to speak for themselves, should be convincing by openly discussing limitations, and by providing extensive risk information. Controversy can be overcome by highlighting potential benefits to public health and to society at-large. People may become more comfortable with $\mathrm{CHI}$ when confronted with them more frequently. $\mathrm{CHI}$ may function as part of the complete pipeline towards licensure.

\section{Ethical perspectives}

Several ethical considerations need to be addressed in order to approve an $\mathrm{CHI}[48,49]$ :

- Are risks and discomforts identified, minimized, determined to be reasonable, and justified by social value?

- Does scientific rationale and design align with social value?

- Is there a system for compensation for research-related injury?

- Are vulnerable populations enrolled?

- Is the informed consent process adequate?

- Is the level of compensation appropriate?

- Can the right to withdraw be respected?

- Will appropriate community engagement occur?

One argument in favor of critically evaluating $\mathrm{CHI}$ for risks is the need to preserve public trust, as risky research can inadvertently undermine longer-term collaboration, e.g. participation in RCTs. The WHO Global Health Ethics Team, as a trusted and neutral partner, 
provides advice on research ethics, including the performance of $\mathrm{CHI}$. One aspect is to build and maintain trust, by increasing accountability, through registries of $\mathrm{CHI}$ studies and associated adverse events. Furthermore, the public and the media should be engaged. Finally, $\mathrm{CHI}$ should lead to benefit sharing and capacity building.

In the evaluation of $\mathrm{CHI}$, several significant gaps remain concerning guidance, data and policy. Guidance is lacking if the consequence of infection is uncertain, and on reasonable limits of risk and how to address these risks to third parties. Aggregating data from multiple studies would improve accuracy and enhance interpretation of potential risk. Finally, with the exception of CHMI [50] and WHO guidance documents [51], there is a lack of guidelines and standards on how to perform $\mathrm{CHI}$. A way forward would be to define a set of principles, covering safety, stewardship, data sharing, capacity strengthening and engagement, to be adopted by members of the $\mathrm{CHI}$ consortium. This consortium can share and exchange information, promote standards and best practices, and develop an infrastructure for ethical data sharing.

Worldwide, ethics committees have a lack of understanding of, and experience with $\mathrm{CHI}$. Capacity building will have to address local needs, as IRBs/ethics committees and regulators need to be prepared, starting with countries where studies are already planned/being performed. A longer-term planning of studies may be helpful to ensure that regulators are well-prepared. A document on the ethics of $\mathrm{CHI}$ will be published in 2018.

In Africa, due to historical atrocities and abuses, there is an overall negative public perception of $\mathrm{CHI}$. Currently, clear ethical guidelines on $\mathrm{CHI}$ evaluation in Africa are absent. The primary goal is to develop a human protection standard equivalent to international 
standards, based on Good Clinical Practices (GCP), GMP, WHO and Council for International Organization of Medical Sciences (CIOMS) guidance and FDA and EMA counterparts. In the long term, harmonization may be achieved within Africa. In contrast with the principle of non-maleficence, $\mathrm{CHI}$ follow the concept of 'do harm to prevent more harm'. To this end, risk minimization and appropriate risk disclosure to volunteers is crucial.

\section{Use of Human Challenge Trials in Developing Countries}

CHMI had not been done in Africa in the modern era due to its reliance on mosquito bite inoculation and the fact that the vector used is Anopheles stephensi, a potent south Asian vector that could pose severe public health risk if introduced. With the development of the PfSPZ Challenge (see above), CHMIs can now be performed safely. Since the first African study in Tanzania [4], 11 more CHMIs have been performed in Tanzania (Jongo, AJTMH, in press, and NCT02613520), Gabon [52], Kenya [53], and NCT02739763), Mali (NCT02627456) and Equatorial Guinea (NCT02859350). CHMIs in the pipeline are an efficacy evaluation of a malaria vaccine in people living with HIV, and testing of herbal products for malaria intervention. CHMIs have enabled African research institutions to play a more pivotal role in the development of malaria drugs and vaccines, an example arguing for accelerated development of $\mathrm{CHI}$ for other diseases in LMIC settings.

Similarly, a controlled human pneumococcal infection model has been set up in Malawi, initially focusing on healthy adults, but at a later stage aiming to enroll people living with HIV. Providing information to potential participants is crucial, and is potentially further complicated in Malawi due to literacy, and limited telephone access. Inclusion in the UK is based (amongst others) on medical history, while medical history is not generally available in 
Malawi. Furthermore, intensive follow-up is necessary, but complicated in Malawi. To overcome these hurdles, the study will be performed on an inpatient basis in Malawi.

Finally, a dengue $\mathrm{CHI}$ is under consideration to be set up in Vietnam. RCTs in Latin America and South-East Asia of a tetravalent, live-attenuated, chimeric dengue vaccine showed poor efficacy, especially in children, and against DENV2 [54]. Furthermore, a weak correlation was observed between neutralizing antibodies and protection. Dengue $\mathrm{CHI}$ might help to evaluate candidate vaccines or therapeutic drugs and provide more insight in the immunity and correlates of protection. Although the local IRB was initially negative, they were more receptive after careful explanation of the potential benefit to society of dengue $\mathrm{CHI}$. Participants should be volunteers, well-informed about the study and risks. While initial studies are scheduled in university students, it would be inappropriate not to enroll rural populations, but they should understand the trial, and receive appropriate payment, using guidelines based on social research.

\section{Use of Human Challenge Trials in Vulnerable Populations}

It is imperative to develop vaccines for children, pregnant women, and immunocompromised. This does not mean, however, that $\mathrm{CHI}$ can be performed in these vulnerable groups. The Declaration of Helsinki states that medical research on a vulnerable group is only justified if the research is responsive to the health needs or priorities of this group and the research cannot be carried out in a non-vulnerable group. The EC regulation $1901 / 2006$ states that medicinal products for the pediatric population should be developed without subjecting this population to unnecessary clinical or other trials. Finally, the WHO $\mathrm{CHI}$ regulatory considerations state that conducting $\mathrm{CHI}$ in children or other vulnerable 
populations would not be deemed acceptable at the present time.

The message from these guidelines is that very careful assessment is needed prior to considering any $\mathrm{CHI}$ in vulnerable populations, with adequate justification an absolute requirement. Infants and young children are the most important target groups for most vaccines. Studies in immunologically experienced adults may not be predictive of effects in immunologically naïve children. Therefore, $\mathrm{CHI}$ could lead to more rapid development of vaccines for important infections in children, shortening timelines and thereby potentially saving lives. However, participation might not offer a direct benefit to the subject; children are unable to give independent informed consent; and parental consent may be vulnerable to coercion. Use of an attenuated pathogen in $\mathrm{CHI}$ in vulnerable groups is an appealing option, especially if already licensed as a vaccine, and this has been done [5]. While such studies may provide evidence of efficacy, and correlates of protection, protection against an attenuated strain may not bridge to protection against the wild type strain, and endpoints are limited to replication of the attenuated pathogen. Other approaches such as field efficacy trials may be feasible and have greater value, especially for infectious agents with high incidence rates.

When field efficacy trials are done in vulnerable populations, high rates of severe adverse events are generally expected and considered acceptable. For example, there were 2318 SAEs in the RTS,S/ASO1b phase 3 trial population ( $N=15,495)$, including 158 deaths [55], despite the availability of standard medical care at each of the 11 study centers. In contrast, a CHMI might involve 20 children, but the likelihood of an SAE, given the extremely close follow-up and short duration of the study, is very small. While a CHMI trial cannot address 
the endpoints of a phase 3 study, it could provide valuable data accelerating the development of life saving products and do so at low risk. The ethical considerations underlying a consideration of CHMI trial in vulnerable populations are therefore complex, and merit ongoing discussion. As an example, after careful review, in which benefits, defined broadly, were considered adequate and risks negligible, the first $\mathrm{CHMI}$ in persons living with HIV has recently been allowed by the FDA and approved by the TFDA and is currently under execution (NCT03420053).

In a completely different approach, self-challenge models in those already chronically infected are possible, for example testing vaccine efficacy in HIV-infected individuals who started anti-retroviral therapy early after infection. Vaccination could be conducted during standard acute treatment interruption, providing information on responses associated with control of viremia or limitation of viral mutations, and could be used to up- or down-select vaccine candidates, and provide insight in the mechanisms of vaccine-induced immunity. By close observation with sensitive techniques, and treatment restart with an HIV RNA increase to levels higher than the pre-treatment viral load, exposure of the volunteer to viremia could be minimized.

\section{Recommendations:}

- When animal models do not exist, and field trials cannot be performed, within reasonable resource constraints, including cost and time, consideration should be given to the conduct of CHI, where the concept of 'do harm to prevent greater harm' is combined with the principle of non-maleficence.

- With respect to the proposed role of $\mathrm{CHI}$ throughout clinical development, a good 
discussion at an early stage between regulator and developer/investigator will streamline the regulatory process, avoiding hurdles at a later stage. This is most important when regulatory guidance does not already exist.

- Most guidelines state that conducting $\mathrm{CHI}$ in children or other vulnerable populations is generally not deemed acceptable at the present time, although each circumstance should be considered carefully. For example, a first CHMI is underway currently in persons living with HIV, after receiving both ethical and regulatory review. One exception to this guidance may be studies where an attenuated pathogen is available to be used as challenge agent, especially if it has been licensed as a vaccine in the target population [5]. However, protection against an attenuated strain may not necessarily lead to protection against the wild type strain.

- As $\mathrm{CHI}$ are generally not performed in children, extrapolating data from adults is essential. Hence, it is necessary to define a $\mathrm{CHI}$ extrapolation plan as part of the study protocol when feasible.

- Further consideration of ethical frameworks in which $\mathrm{CHI}$ might be able to be performed in children are needed, as extrapolation may not be possible due to preexisting immunity in adults vs. unexposed non-immune children who require protection from the disease under study. Furthermore, correlates of protection may differ between different age groups. Consideration of balancing the risks associated with a $\mathrm{CHI}$ to the risks associated with a large, lengthy field trial might provide one such reflection.

- $\quad \mathrm{CHI}$, similar to field trials, generate precious samples; a systems biology approach may be useful, using 'omics' technologies to investigate the samples to the full, to 
obtain a better understanding of pathogenicity, to drive diagnostic and therapeutic development, and to find correlates of protection.

- To identify and isolate a new challenge agent, relevant source populations need to be determined. Established, predominant or circulating strains will offer greater relevance to study data than minority or uncharacteristically resistant serotypes, whereas newly emergent, pandemic strains may offer unacceptable risks of adverse events or sequelae, including a high case fatality rate for conditions that cannot be treated.

- The same quality criteria should apply to the challenge strain, as for any other substance administered to individuals in a clinical trial setting. Adequate efforts should be made for the characterization, qualification and identification of the critical quality attributes to be met, so as to get reliable and consistent challenge material and relevant clinical information.

- To be optimally prepared for an audit visit, $\mathrm{CHI}$ sites should develop a clear and comprehensive QC plan, data management plan and ensure that quality records are available at all times.

- As CHI can only be performed if volunteers are available, it is worthwhile to invest in improving the participant's experience, looking at motivation, study experience, and satisfaction with reimbursement level.

- Aggregating data from multiple studies would make the data stronger and enhance interpretation. This can only be achieved if procedures and models are standardized where possible.

- Communication to the public is of major importance to gain support for $\mathrm{CHI}$, as well as to help recruitment of volunteers. To this end, the studies need to be properly 
explained, including the risks, in a balanced way. Information material for lay audience or volunteers may prove helpful.

- To increase $\mathrm{CHI}$ capacity, the units should not get too big, as building relationships with volunteers is important, but also for safety of volunteers and staff; smaller studies are easier to perform. Therefore, more centers are needed, not necessarily bigger centers.

In sum, this conference provided a good overview of the current state of the art, and directions for further action towards the next conference.

\section{Acknowledgements}

The authors would like to thank Aaron Mendelsohn (P95, Epidemiology and Pharmacovigilance Consulting and Services) for careful review of this paper.

\section{Funding}

This research did not receive any specific grant from funding agencies in the public, commercial, or not-for-profit sectors.

\section{Declaration of interest}

Conflict of interest statement: T.L.R., is a salaried employee of Sanaria Inc., the developer and owner of PfSPZ Vaccine and PfSPZ Challenge. The other authors declare no conflict of interest.

\section{References}

[1] Sheets RL, Fritzell B, Aguado de Ros MT. Human challenge trials in vaccine development: Strasbourg, September 29 - October 1, 2014. Biologicals. 2016;44:37-50. [2] The National Commission for the Protection of Human Subjects of Biomedical and Behavioral Research. The Belmont Report - Ethical Principles and Guidelines for the Protection of Human Subjects of Research. US Department of Health \& Human Services; 1979.

[3] Food and Drug Administration (FDA). IRB Responsibilities for Reviewing the Qualifications of Investigators, Adequacy of Research Sites, and the Determination of Whether an IND/IDE is Needed. 2013.

[4] Shekalaghe S, Rutaihwa M, Billingsley PF, Chemba M, Daubenberger CA, James ER, et al. Controlled human malaria infection of Tanzanians by intradermal injection of aseptic, 
purified, cryopreserved Plasmodium falciparum sporozoites. The American journal of tropical medicine and hygiene. 2014;91:471-80.

[5] Groome MJ, Koen A, Fix A, Page N, Jose L, Madhi SA, et al. Safety and immunogenicity of a parenteral P2-VP8-P[8] subunit rotavirus vaccine in toddlers and infants in South Africa: a randomised, double-blind, placebo-controlled trial. The Lancet Infectious diseases.

2017;17:843-53.

[6] Epstein JE, Paolino KM, Richie TL, Sedegah M, Singer A, Ruben AJ, et al. Protection against Plasmodium falciparum malaria by PfSPZ Vaccine. JCI Insight. 2017;2:e89154.

[7] Persson C, Oliveira GA, Sultan AA, Bhanot P, Nussenzweig V, Nardin E. Cutting edge: a new tool to evaluate human pre-erythrocytic malaria vaccines: rodent parasites bearing a hybrid Plasmodium falciparum circumsporozoite protein. Journal of immunology (Baltimore, Md : 1950). 2002;169:6681-5.

[8] Espinosa DA, Yadava A, Angov E, Maurizio PL, Ockenhouse CF, Zavala F. Development of a chimeric Plasmodium berghei strain expressing the repeat region of the P. vivax circumsporozoite protein for in vivo evaluation of vaccine efficacy. Infection and immunity. 2013;81:2882-7.

[9] Kaba SA, Karch CP, Seth L, Ferlez KMB, Storme CK, Pesavento DM, et al. Selfassembling protein nanoparticles with built-in flagellin domains increases protective efficacy of a Plasmodium falciparum based vaccine. Vaccine. 2017.

[10] Espinosa DA, Gutierrez GM, Rojas-Lopez M, Noe AR, Shi L, Tse SW, et al. Proteolytic Cleavage of the Plasmodium falciparum Circumsporozoite Protein Is a Target of Protective Antibodies. J Infect Dis. 2015;212:1111-9.

[11] Espinosa DA, Radtke AJ, Zavala F. Development and Assessment of Transgenic Rodent Parasites for the Preclinical Evaluation of Malaria Vaccines. Methods in molecular biology (Clifton, NJ). 2016;1403:583-601.

[12] Robertson JS, Bootman JS, Newman R, Oxford JS, Daniels RS, Webster RG, et al. Structural changes in the haemagglutinin which accompany egg adaptation of an influenza A(H1N1) virus. Virology. 1987;160:31-7.

[13] Robertson JS, Naeve CW, Webster RG, Bootman JS, Newman R, Schild GC. Alterations in the hemagglutinin associated with adaptation of influenza B virus to growth in eggs. Virology. 1985;143:166-74.

[14] van Baalen CA, Els C, Sprong L, van Beek R, van der Vries E, Osterhaus AD, et al. Detection of nonhemagglutinating influenza a(h3) viruses by enzyme-linked immunosorbent assay in quantitative influenza virus culture. Journal of clinical microbiology. 2014;52:16727.

[15] Kirkpatrick BD, Whitehead SS, Pierce KK, Tibery CM, Grier PL, Hynes NA, et al. The live attenuated dengue vaccine TV003 elicits complete protection against dengue in a human challenge model. Science translational medicine. 2016;8:330ra36.

[16] Minassian AM, Ronan EO, Poyntz H, Hill AV, McShane H. Preclinical development of an in vivo BCG challenge model for testing candidate TB vaccine efficacy. PloS one. 2011;6:e19840.

[17] Villarreal-Ramos B, Berg S, Chamberlain L, McShane H, Hewinson RG, Clifford D, et al. Development of a BCG challenge model for the testing of vaccine candidates against tuberculosis in cattle. Vaccine. 2014;32:5645-9.

[18] Harris SA, Meyer J, Satti I, Marsay L, Poulton ID, Tanner R, et al. Evaluation of a human BCG challenge model to assess antimycobacterial immunity induced by BCG and a candidate tuberculosis vaccine, MVA85A, alone and in combination. J Infect Dis.

2014;209:1259-68. 
[19] Fix AD, Harro C, McNeal M, Dally L, Flores J, Robertson G, et al. Safety and immunogenicity of a parenterally administered rotavirus VP8 subunit vaccine in healthy adults. Vaccine. 2015;33:3766-72.

[20] Panchaud A, Stojanov M, Ammerdorffer A, Vouga M, Baud D. Emerging Role of Zika Virus in Adverse Fetal and Neonatal Outcomes. Clinical microbiology reviews. 2016;29:65994.

[21] Shah S, Kimmelman J, Drapkin Lyerly A, Fernandez Lynch H, McCutchan F, Miller F, et al. Ethical considerations for Zika virus human challenge trials. 2017.

[22] Yeung KHT, Duclos P, Nelson EAS, Hutubessy RCW. An update of the global burden of pertussis in children younger than 5 years: a modelling study. The Lancet Infectious diseases. 2017;17:974-80.

[23] Harro C, Chakraborty S, Feller A, DeNearing B, Cage A, Ram M, et al. Refinement of a human challenge model for evaluation of enterotoxigenic Escherichia coli vaccines. Clinical and vaccine immunology : CVI. 2011;18:1719-27.

[24] Porter CK, Riddle MS, Alcala AN, Sack DA, Harro C, Chakraborty S, et al. An Evidenced-Based Scale of Disease Severity following Human Challenge with Enteroxigenic Escherichia coli. PloS one. 2016;11:e0149358.

[25] Chen WH, Cohen MB, Kirkpatrick BD, Brady RC, Galloway D, Gurwith M, et al. Single-dose Live Oral Cholera Vaccine CVD 103-HgR Protects Against Human Experimental Infection With Vibrio cholerae O1 El Tor. Clinical infectious diseases : an official publication of the Infectious Diseases Society of America. 2016;62:1329-35.

[26] Lyke KE, Ishizuka AS, Berry AA, Chakravarty S, DeZure A, Enama ME, et al. Attenuated PfSPZ Vaccine induces strain-transcending T cells and durable protection against heterologous controlled human malaria infection. Proc Natl Acad Sci U S A. 2017;114:27116.

[27] Atmar RL, Bernstein DI, Harro CD, Al-Ibrahim MS, Chen WH, Ferreira J, et al. Norovirus vaccine against experimental human Norwalk Virus illness. N Engl J Med. 2011;365:2178-87.

[28] Bernstein DI, Atmar RL, Lyon GM, Treanor JJ, Chen WH, Jiang X, et al. Norovirus vaccine against experimental human GII.4 virus illness: a challenge study in healthy adults. J Infect Dis. 2015;211:870-8.

[29] Steffen R, Hill DR, DuPont HL. Traveler's diarrhea: a clinical review. Jama. 2015;313:71-80.

[30] Riddle MS, Sanders JW, Putnam SD, Tribble DR. Incidence, etiology, and impact of diarrhea among long-term travelers (US military and similar populations): a systematic review. The American journal of tropical medicine and hygiene. 2006;74:891-900. [31] Porter CK, Thura N, Ranallo RT, Riddle MS. The Shigella human challenge model. Epidemiol Infect. 2013;141:223-32.

[32] Anwar E, ;, Goldberg E, Fraser A, Acosta C, Paul M, Leibovici L. Vaccines for preventing typhoid fever. . Cochrane Database of Systematic Reviews 2014.

[33] Waddington CS, Darton TC, Jones C, Haworth K, Peters A, John T, et al. An outpatient, ambulant-design, controlled human infection model using escalating doses of Salmonella Typhi challenge delivered in sodium bicarbonate solution. Clinical infectious diseases : an official publication of the Infectious Diseases Society of America. 2014;58:1230-40.

[34] Darton TC, Jones C, Blohmke CJ, Waddington CS, Zhou L, Peters A, et al. Using a Human Challenge Model of Infection to Measure Vaccine Efficacy: A Randomised, Controlled Trial Comparing the Typhoid Vaccines M01ZH09 with Placebo and Ty21a. PLoS neglected tropical diseases. 2016;10:e0004926.

[35] Juel HB, Thomaides-Brears HB, Darton TC, Jones C, Jones E, Shrestha S, et al. Salmonella Typhi Bactericidal Antibodies Reduce Disease Severity but Do Not Protect 
against Typhoid Fever in a Controlled Human Infection Model. Frontiers in immunology. 2017;8:1916.

[36] Shi T, McAllister DA, O'Brien KL, Simoes EAF, Madhi SA, Gessner BD, et al. Global, regional, and national disease burden estimates of acute lower respiratory infections due to respiratory syncytial virus in young children in 2015: a systematic review and modelling study. Lancet (London, England). 2017;390:946-58.

[37] Habibi MS, Jozwik A, Makris S, Dunning J, Paras A, DeVincenzo JP, et al. Impaired Antibody-mediated Protection and Defective IgA B-Cell Memory in Experimental Infection of Adults with Respiratory Syncytial Virus. American journal of respiratory and critical care medicine. 2015;191:1040-9.

[38] Frenck R, Bernstein DI, Xia M, Huang P, Zhong W, Parker S, et al. Predicting susceptibility to norovirus GII.4 by use of a challenge model involving humans. J Infect Dis. 2012;206:1386-93.

[39] Ferreira DM, Neill DR, Bangert M, Gritzfeld JF, Green N, Wright AK, et al. Controlled human infection and rechallenge with Streptococcus pneumoniae reveals the protective efficacy of carriage in healthy adults. American journal of respiratory and critical care medicine. 2013;187:855-64.

[40] Pennington SH, Pojar S, Mitsi E, Gritzfeld JF, Nikolaou E, Solorzano C, et al. Polysaccharide-Specific Memory B Cells Predict Protection against Experimental Human Pneumococcal Carriage. American journal of respiratory and critical care medicine. 2016;194:1523-31.

[41] Mitsi E, Roche AM, Reine J, Zangari T, Owugha JT, Pennington SH, et al. Agglutination by anti-capsular polysaccharide antibody is associated with protection against experimental human pneumococcal carriage. Mucosal immunology. 2017;10:385-94.

[42] Mahmoudi S, Keshavarz H. Efficacy of phase 3 trial of RTS, S/AS01 malaria vaccine: The need for an alternative development plan. Human vaccines \& immunotherapeutics. 2017;13:2098-101.

[43] van den Hoogen BG, de Jong JC, Groen J, Kuiken T, de Groot R, Fouchier RA, et al. A newly discovered human pneumovirus isolated from young children with respiratory tract disease. Nature medicine. 2001;7:719-24.

[44] Biacchesi S, Skiadopoulos MH, Tran KC, Murphy BR, Collins PL, Buchholz UJ. Recovery of human metapneumovirus from cDNA: optimization of growth in vitro and expression of additional genes. Virology. 2004;321:247-59.

[45] Biacchesi S, Murphy BR, Collins PL, Buchholz UJ. Frequent frameshift and point mutations in the SH gene of human metapneumovirus passaged in vitro. Journal of virology. 2007;81:6057-67.

[46] Talaat KR, Karron RA, Thumar B, McMahon BA, Schmidt AC, Collins PL, et al. Experimental infection of adults with recombinant wild-type human metapneumovirus. J Infect Dis. 2013;208:1669-78.

[47] Darton TC, Blohmke CJ, Moorthy VS, Altmann DM, Hayden FG, Clutterbuck EA, et al. Design, recruitment, and microbiological considerations in human challenge studies. The Lancet Infectious diseases. 2015;15:840-51.

[48] Miller FG, Grady C. The ethical challenge of infection-inducing challenge experiments. Clinical infectious diseases : an official publication of the Infectious Diseases Society of America. 2001;33:1028-33.

[49] Bambery B, Selgelid M, Weijer C, Savulescu J, Pollard A. Ethical Criteria for Human Challenge Studies in Infectious Diseases. Public Health Ethics. 2016;9:92-103.

[50] Laurens MB, Duncan CJ, Epstein JE, Hill AV, Komisar JL, Lyke KE, et al. A consultation on the optimization of controlled human malaria infection by mosquito bite for evaluation of candidate malaria vaccines. Vaccine. 2012;30:5302-4. 
[51] EXPERT COMMITTEE ON BIOLOGICAL STANDARDIZATION. Human Challenge Trials for Vaccine Development: regulatory considerations. In: WHO, editor. Geneva, Switzerland2016.

[52] Lell B, Mordmuller B, Dejon Agobe JC, Honkpehedji J, Zinsou J, Mengue JB, et al. Impact of Sickle Cell Trait and Naturally Acquired Immunity on Uncomplicated Malaria after Controlled Human Malaria Infection in Adults in Gabon. The American journal of tropical medicine and hygiene. 2018;98:508-15.

[53] Hodgson SH, Juma E, Salim A, Magiri C, Kimani D, Njenga D, et al. Evaluating controlled human malaria infection in Kenyan adults with varying degrees of prior exposure to Plasmodium falciparum using sporozoites administered by intramuscular injection. Frontiers in microbiology. 2014;5:686.

[54] Wilder-Smith A, Massad E. Age specific differences in efficacy and safety for the CYDtetravalent dengue vaccine. Expert review of vaccines. 2016;15:437-41.

[55] RTS SCTP. Efficacy and safety of RTS,S/AS01 malaria vaccine with or without a booster dose in infants and children in Africa: final results of a phase 3, individually randomised, controlled trial. Lancet (London, England). 2015;386:31-45. 